\title{
Relayer Selection Strategies in Cellular Networks with Peer-to-Peer Relaying
}

\author{
V. Sreng, H. Yanikomeroglu, and D. D. Falconer \\ Broadband Communications and Wireless Systems (BCWS) Centre \\ Dept. of Systems and Computer Engineering \\ Carleton University, Ottawa, Canada \\ \{vsreng,halim,ddf\}@ sce.carleton.ca
}

\begin{abstract}
We consider a TDMA cellular multihop network where relaying - via wireless terminals that have a good communication link to the base station - is used as a coverage enhancement technique. Provided that the subscriber density is not very low, relaying vi a wireless terminals can have a significant impact on coverage, capacity, and throughput. This is mainly due to the fact that the signals only have to travel through shorter distances and/or improved paths. In this work, we investigated the effects of relaying node selection strategies (essentially a routing issue) and maximum relayer transmit power level on coverage. Our simulation results show that with a very modest level of relaying node transmit power and with some moderate intelligence incorporated in the relaying node selection scheme, the (high data rate) coverage can be improved significantly through two-hop relaying without consuming any additional bandwidth.
\end{abstract}

\section{INTRODUCTION}

Peer-to-peer relaying technology originates in packet radio and ad-hoc networks where the traffic signals have to be relayed through one or more intermediate node(s), depending on the distance between the source and the destination nodes [1], [2].

Using relaying - via mobile terminals - as a coverage enhancement technique in a cellular network is a fairly novel concept, and is gaining a strong interest [3] and consideration in the research community such as the WWRF (World Wireless Research Forum) [4].

In [5] we presented a comparison of channel selection methods for relaying purposes, along with just one pathlossbased relaying node selection strategy. A major finding in that paper was that the coverage improvement obtained from relaying is rather insensitive to the relaying channel selection strategy provided that some diligence is shown in relaying node selection and power control is applied. In this paper, we investigate the sensitivity of relaying systems to relaying node selection (routing) and relaying node maximum power level, which were not covered in [5].

Basically two forms of relaying can be carried out - analog form or digital form. In the analog form of relaying, the signals received are forwarded without any signal processing; hence, as the signal is amplified, so is the noise. In the digital form, on the other hand, the received signals are first demodulated and detected, and thus cleaned from noise and interference (as much as possible), and then re-encoded and re-modulated before forwarding; this process is sometimes referred to as "decode-and-forward relaying" [6],[7] or "regenerative relaying" [8]. Thus, when digital form of relaying is employed, there is no noise propagation from one hop to the next; however there is a danger of detection error propagation [6],[7].

It should be borne in mind that for delay sensitive traffic, such as voice, the digital form of relaying may not be suitable due to the incurred delay. Meanwhile, other traffic types, which are less sensitive to delay and which require large bandwidth to accommodate high data rates, would require the digital form of relaying in order to prevent the noise propagation and thus to minimize the error rate. We consider only the digital form of relaying in this paper.

Routing is very crucial and challenging in ad hoc networks. Routing protocols must be power-aware, robust, and resilient to changes in the network topology; moreover, these protocols should achieve all of these goals without excessive overhead. Routing in cellular multihop networks, on the other hand, is likely to be much more manageable, mainly due to the presence of a central node (which is the common source in the downlink and the common destination in the uplink) with much more functionality and intelligence, namely, the base station. Nevertheless, routing (relaying node selection) is still a non-trivial issue in cellular multihop networks since there will often be many candidate relaying nodes for a node ${ }^{1}$ that requires relaying assistance and not choosing the optimal relaying node can have potential impacts on the overall performance improvement.

Since power is a very precious entity at the wireless terminals, the power allocated for relaying other terminals' signals is an important parameter for the relaying terminals.

In this paper we only consider the downlink in two-hop cellular networks; mobility is not taken into account.

\section{RELAYING NODE AND RELAYING CHANNEL SELECTION SCHEMES}

In this paper, we consider a relaying channel selection scheme based on the maximum SIR, which we referred to in [5] as the Semi-Smart Channel Selection. In our modeling, first the relaying node selection is performed for each node

\footnotetext{
${ }^{1}$ In this paper, the terms "node", "wireless terminal", and "subscriber", are used interchangeably.
} 
that requires relaying assistance, and the relaying channel selection process then follows (i.e., the two selection processes are sequential).

Choosing a relaying node solely based on the physical distance will not be as effective as choosing one based on the pathloss (because of the shadowing effects); however, location-based relaying node selection schemes are attractive as they can be carried out quite simply with the aid of location techniques. In fact, location-based routing has already been proposed for ad hoc networks in order to reduce the routing overhead and to maintain a small routing table at the mobile nodes [9]-[11]. Our results for the location-based relaying node selection schemes are somewhat optimistic, since we assume perfect knowledge of location.

In this paper, the small-scale multipath fading effects are excluded during the relaying node and channel selection processes since it would be impractical to perform interrelaying node and inter-relaying channel hand-offs based on multipath conditions. However, after the relaying node and channel are selected, the effects of multipath fading are included in the coverage simulations.

\section{A. Relaying Node Selection}

\section{A.1 Selection Based on Physical Distance}

Let $N$ denote the set of candidate relaying nodes defined as the nodes which have an adequate link to the base station in a two-hop relaying network (see (9) for the definition of adequate link). Let $d_{n 1}$ and $d_{n 2}$ be the distances associated with the first (between the base station to the candidate relaying node) and the second (between the candidate relaying node to the relayed node) hops, respectively, along the $n^{\text {th }}$ route, where $n \in N$.

Then, the selected route, $r_{s}$, is determined as follows in each selection scheme:

- Shortest Total Distance (STD) Selection:

$$
r_{s}=\arg \min _{\text {all }}\left(d_{n \in N}+d_{n 2}\right)
$$

- Least Longest Hop (LLH) Selection:

$$
r_{s}=\arg \min _{\text {all } n \in N}\left(\max \left(d_{n 1}, d_{n 2}\right)\right)
$$

- Shortest Relaying Hop Distance (SRD) Selection:

$$
r_{s}=\arg \min _{\text {all } n \in N}\left(d_{n 2}\right)
$$

\section{A.2 Selection Based on Pathloss}

As discussed earlier, although it is expected that a relaying node selection based on pathloss will be superior to the one based solely on distance, the pathloss-based selection will incur higher signaling overhead (mainly due the pathloss estimation techniques which are not considered in this paper); this disadvantage must be weighted against the additional performance return.

In the following, we investigate three relaying node selection schemes in a two-hop relaying network: the first is a scheme that makes the selection based on the least pathloss in the two combined hops; the second is based on a route that has the lowest bottleneck (in terms of pathloss); and the third is based on the least pathloss in only the second hop (which we call the relaying hop).

Similar to the notations used in the previous section, let $P L_{n 1}$ and $P L_{n 2}$ denote the pathlosses in $\mathrm{dB}$ associated with the first and the second hop, respectively, along the $n^{\text {th }}$ route, where $n \in N$. Then, the selected route, $r_{s}$, is determined as follows in each selection scheme:

- Minimum Total Pathloss (MTP) Selection:

$$
r_{s}=\arg \min _{\text {all } n \in N}\left(P L_{n 1}+P L_{n 2}\right)
$$

- Least Maximum Pathloss (LMP) Selection (used in [3]):

$$
r_{s}=\arg \min _{\text {all }}\left(\max \left(P L_{n 1}, P L_{n 2}\right)\right)
$$

- Minimum Relaying Hop Pathloss (MRP) Selection:

$$
r_{s}=\arg \min _{\text {all } n \in N}\left\{P L_{n 2}\right\}
$$

Fig. 1 illustrates a cell with a base station, a relayed node, and three candidate relaying nodes. Associated with each candidate relaying node, there is a candidate path (route), marked as I, II, and III in Fig. 1. Path I corresponds to the minimum relaying hop pathloss selection, path II corresponds to the minimum total pathloss selection, and path III corresponds to the least maximum pathloss selection scheme. There will be as many candidate paths (routes) as the number of nodes that have a good link with the base station (i.e., candidate relaying nodes). In practice, however, each node that requires relaying assistance should limit its candidate relaying nodes set to only a few in order to minimize the routing overhead.

\section{B. Channel Selection}

Once a relaying node has been determined, an additional channel must be assigned for the relaying hop link to prevent feedback loops. Based on our previous paper [5], we propose reusing channels from the adjacent cells for relaying purposes; the very appealing advantage of this strategy is that no additional bandwidth is consumed in the relaying mode. One of the channel selection schemes described in [5] is reintroduced here for convenience. Let $\gamma_{i}^{c}$ be the signal-tointerference ratio (SIR) experienced at the relayed node $i$, on channel $c$, and $B_{c}$ the set of all base stations that use channel $c$ plus the relay node $j$ which will also use channel $c$ for relaying purposes (i.e., $B_{c}$ is the augmented co-channel set for channel $c$ ). Then,

$$
\gamma_{i}^{c}=\frac{G_{j i} P_{j}^{c}}{\sum_{k \in B_{c}, k \neq j} G_{k i} P_{k}^{c}},
$$

where $G_{j i}$ is the pathloss between the relayed node $i$ and the relaying node $j$, and $P_{j}^{c}$ is the transmitted power of the relaying node $j$. Similarly, $G_{k i}$ is the pathloss between the 
relayed node $i$ and a co-channel base station $k$ whose channel is being probed for reuse, and $P_{k}^{c}$ is the corresponding base station transmit power. Also, let $L$ denote the set of all channels in the adjacent cells (within the cluster of the subscriber which needs relaying assistance). Then, a channel, $l_{s}$, is selected as follows:

- Maximum SIR Selection:

$l_{s}=\arg \max _{\text {allc } \in L}\left(\gamma_{i}^{c}\right)$

\section{SIMULATION MODEL}

We consider the downlink of a TDMA urban cellular network where two-hop relaying is used whenever there does not exist a sufficiently good direct link between a base station and a wireless terminal.

Our propagation model consists of distance-dependent attenuation with a propagation exponent of 4, lognormal shadowing (standard deviation $10 \mathrm{~dB}$ ), and flat Rayleigh fading. The same pathloss model is used between a base station and a wireless terminal, and between one wireless terminal and another. The simulation area consists of $6 \times 6$ square cells with wrap around edges. The cluster size is chosen to be 4 . Only the $400 \times 400$ meters cell size is considered in this paper, which represents an interferencelimited environment. Omnidirectional antennas are used at both base stations and wireless terminals.

The carrier frequency is taken to be $2.5 \mathrm{GHz}$ and the transmission bandwidth $2 \mathrm{MHz}$. The thermal noise is also considered at the receiver, with a noise figure of $8 \mathrm{~dB}$. Due to the large bandwidth and noise figure assumptions, the digital form of relaying is used throughout. We considered only twohop relaying with single-class traffic where every wireless terminal has the same SINR requirement of $10 \mathrm{~dB}$ (this rather high value for SINR may correspond to a particular constellation in a system employing adaptive modulation and coding).

Simulations are run with the incorporation of transmit power control. Whenever power control is employed, a "snapshot" scheme is used for both relaying and non-relaying cases with a step size of $2 \mathrm{~dB}$. Power updates are performed until the receiver's SINR falls between 10 and $12 \mathrm{~dB}$, or until a maximum of 10 updates are reached, whichever comes first.

The nodes within a cell are placed randomly according to a two-dimensional uniform distribution. The number of channels available in each cell is assumed to be equal to the number of (active) wireless terminals; that is, all the cells are assumed to be fully loaded before relaying, and therefore, a relaying channel has to be chosen among the already used channels in the adjacent cells. The simulation process consists of the following steps:

1) Set the number of subscribers (nodes) per cell, $S$.

2) Place the subscribers randomly across the network while maintaining $S$ subscribers per cell. Each subscriber is given service through the closest base station. Assign an independent lognormal shadowing component between each subscriber and its base station.

3) Determine whether each subscriber has a sufficiently good link with its base station or not. Towards that end, collect 100 SINR samples for each subscriber with each sample undergoing independent Rayleigh fading (note that all of the 100 samples have the same lognormal shadowing component). Execute a snapshot power control before recording each SINR sample. For a given subscriber, if $95 \%$ or more SINR samples turn out to be above the $10 \mathrm{~dB}$ threshold, i.e., if $\operatorname{Pr}[\operatorname{SINR} \geq 10 \mathrm{~dB}] \geq 95 \%$,

then classify that subscriber as one having a sufficiently good link with its base station (such a subscriber does not require relaying; indeed, it constitutes a candidate relaying node for those subscribers which need relaying assistance). Otherwise (i.e., if the complement of (9) is true), classify it as a subscriber that has a poor link (and therefore that subscriber will require relaying assistance).

4) For all subscribers that require relaying assistance, choose a relaying node and a relaying channel according to the algorithms specified in Section II (note that the multipath fading is not taken into account in both relaying node and relaying channel selection schemes).

5) With relaying incorporated, collect once again 100 SINR samples for each subscriber, incorporating independent Rayleigh fading in each sample, also incorporating snapshot power control for each sample. For subscribers which are served by their base stations through a single-hop, use (11) to determine whether the links are sufficiently good; and for those subscribers whose signals are delivered in two-hops, use the following criterion:

$\operatorname{Pr}\left[\left\{S I N R_{1} \& S I N R_{2}\right\} \geq 10 \mathrm{~dB}\right] \geq 95 \%$.

In the above, subscripts 1 and 2 correspond to the first and second hop, respectively. In the SINR calculation, take into account the interference created as a result of relaying (an independent Rayleigh fading and a lognormal shadowing component are associated with all the interference links).

Record the number of subscribers that have good links, according to (9) or (10) as appropriate, only in the four cells that constitute the innermost cluster in the 9-cluster wrap around network.

6) Repeat steps 2)-5) for a total of 1000 times. The fraction of subscribers that have good links, out of the total number of 4 (number of cells in the innermost cluster) x $S$ (number of subscribers per cell) x 1000 (number of runs) subscribers observed, yields the desired coverage value.

7) Go to step 1) and repeat steps 1)-6) for various $S$ values. 


\section{SIMULATION RESULTS}

For the results shown below, the maximum base station transmit power used for any mobile node is $1 \mathrm{~W}(30 \mathrm{dBm})$. From Fig. 2, due to the shadowing effect we observe that the relayer selection schemes based on pathloss offer a consistently superior performance in comparison to those based on distance alone, as expected. It is worthy to note the performance difference among the three selection schemes. For the distance-based relayer selection schemes, the enhanced coverage ranges from approximately $76 \%$ to $90 \%$, at a subscriber density of 64 and a maximum relaying node transmit power of $20 \mathrm{~mW}$, over the $70 \%$ case with no relaying. Meanwhile, for the pathloss-based selection schemes, we see an even larger performance difference among the three schemes. For instance, the highest coverage improvement is approximately 97\%, which is based on the Least Maximum Pathloss (LMP) scheme; meanwhile, the least improvement, approximately $79 \%$, is based on the Minimum Total Pathloss (MTP) scheme.

Finally, Fig. 3 offers a perspective on how the subscriber density impacts the range of maximum relaying node transmit power levels required to achieve a maximum attainable coverage improvement at that particular subscriber density. As can be observed, at a low subscriber density (16 and below) we see that the cut off maximum relaying node transmit power is approximately $30 \mathrm{dBm}(1 \mathrm{~W})$ before the performance improvement starts to saturate; while, at the high subscriber density (32 and above), the cut off is approximately $24.8 \mathrm{dBm}(300 \mathrm{~mW})$. What this reveals is that at a reasonably high subscriber density, most of the potential coverage improvement can be obtained at fairly low relaying node maximum transmit power levels.

\section{DISCUSSIONS AND CONCLUSIONS}

In this paper we investigated the impact of various relaying node selection strategies on the system coverage in a fully loaded cellular system. Our results show that coverage is sensitive to the relaying node selection strategy. Moreover, the degree of improvement depends on the density and maximum transmit power level of potential relaying nodes. In small cells (interference-limited system), the coverage enhancement due to relaying can be increased significantly (particularly when using pathloss-based selection schemes) while using only a low relaying node transmit power. It should be noted that this dramatic increase is obtained with the use of power control. Moreover, this performance improvement is obtained even without reserving extra channels for relaying purposes, since the additional interference due to relaying is confined to within the vicinity of the relaying/relayed node pair as a result of power control.

Finally, based on the results in Fig. 3 it can be concluded that the higher the subscriber density, the less transmit power it is required of the relaying terminals since a reasonably good relaying node selection scheme can be applied in order to assign the best relaying/relayed node pair, which will in turn have a positive effect on the coverage improvement.

A similar work is reported in [12] in which "ODMA" (opportunity-driven multiple access) type of relaying is considered in a CDMA network to increase the coverage for voice communications. Although a similar finding to ours is observed there (i.e., coverage is significantly improved with relaying), it should be noted that the power control algorithm and the signaling protocol required are, in general, more stringent and complex for CDMA-based relaying network in comparison to TDMA-based types; this is mainly due to the inherent interference-limited nature of CDMA networks (we also tried to make our simulation network as interferencelimited as possible by assuming fully loaded cells). More information on signaling and routing protocols required to perform relaying in a cellular network can be found in [12].

Other issues that still remain open include relaying through fixed relayers versus wireless terminals (peer-to-peer relaying), two-hop versus multihop relaying, and mobility and handoff management in peer-to-peer relaying networks. The advantages of using fixed relayers include not consuming precious resources of the wireless terminals (which would be the case in peer-to-peer relaying), less handoffs in a mobile environment, minimum service guarantee (finding a relayer will become more difficult in an unloaded peer-to-peer relaying network), and better security. The main disadvantage of fixed relayers, on the other hand, is the deployment cost. It is likely that future cellular networks may support relaying through both fixed relayers as well as wireless terminals.

One final issue worth noting is that peer-to-peer relaying does not work properly in the FDD (frequency-division duplex) mode with the current wireless terminal technology. This is due to the fact in the FDD mode, the wireless terminals transmit and received at two different frequency bands, although a relaying wireless terminal has to be able to transmit and receive in both bands. Therefore, TDD (timedivision duplex) is a more appropriate mode for peer-to-peer relaying. Since fixed relayers may potentially be equipped with more advanced hardware and signal processing techniques, their operation in the FDD mode may be possible.

\section{ACKNOWLEDGMENT}

This research was supported by a grant from Nortel Networks, Ottawa. In particular, the authors would like to thank to Dr. Bassam Hashem (for pointing out the locationbased technology as an aid in simplifying routing) and Dr. Shalini Periyalwar of Nortel Networks.

\section{REFERENCES}

[1] C. Chang and J. Chang, "Optimal design parameters in a multihop packet radio network using random access techniques," IEEE Globecom, vol. 1, pp. 493-497, 1984.

[2] E. Royer, "A review of current routing protocols for ad hoc mobile wireless networks," IEEE Personal Communications, pp. 46-55, April 1999. 
[3] H. Yanikomeroglu, "Fixed and mobile relaying technologies for cellular networks", Second Workshop on Applications and Services in Wireless Networks (ASWN'02), pp. 75-81, July 2002, Paris, France.

[4] World Wireless Research Forum (WWRF) website, http://www.wireless-world-research.org

[5] V. Sreng, H. Yanikomeroglu, D. Falconer, "Coverage enhancement through two-hop relaying in cellular radio systems," IEEE Wireless Commun. and Networking Conf. (WCNC'02), March 2002, Orlando, USA.

[6] J. Laneman and G. Wornel, "Energy efficient antenna sharing and relaying for wireless networks," IEEE Wireless Commun. and Networking Conf. (WCNC'00), pp. 7-12, Sept. 2000, Chicago, USA.

[7] J. Boyer, D. D. Falconer, and H. Yanikomeroglu, "A theoretical characterization of the multihop wireless communications channel with diversity," IEEE Globecom, Nov. 2001, San Antonio, USA.

[8] M. Hasna and M. Alouini, "End-to-end performance of transmission systems with relays over Rayleigh fading channels," submitted to IEEE Transactions on Wireless Communications.

[9] Y. Ko and N. Vaidya, "Location-aided routing (LAR) in mobile ad hoc networks," MobiCom, pp. 66-74, 1998.

[10] R. Jain, et. al, "Geographical routing using partial information for wireless ad hoc networks," IEEE Communications Magazine, pp. 48-57, Feb. 2001.

[11] I. Stojmenovic, "Position-based routing in ad hoc networks", IEEE Communications Magazine, pp.128134, July 2002.

[12] T. Rouse, I. Band, and S. McLaughlin, "Capacity and power investigation of opportunity driven multiple access (ODMA) networks in TDD-CDMA based systems," IEEE Int.'l Conf. on Commun. (ICC'02), pp. 3202-3206, April 2002, New York, USA.

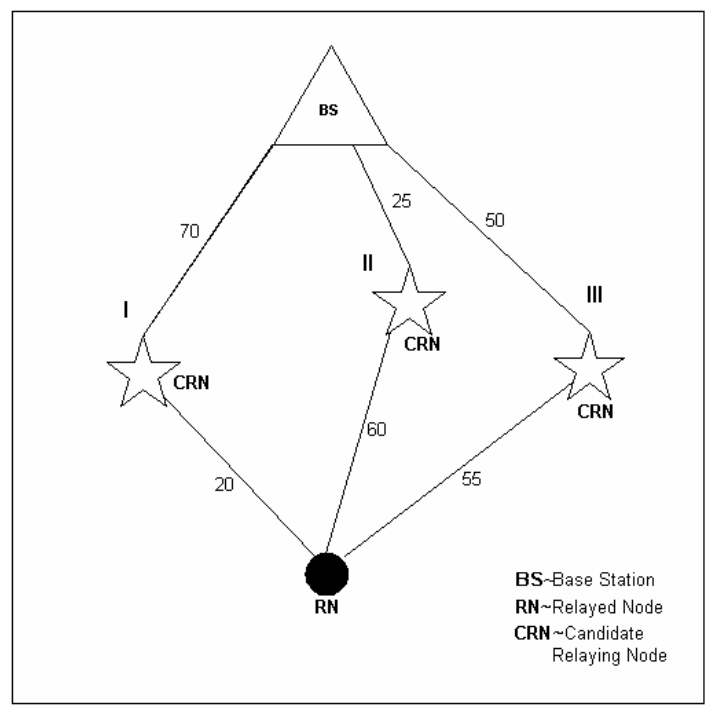

Fig. 1. Illustration of three possible routes based on three different relaying node selection schemes (the numbers attached to the edges indicate the pathlosses in $\mathrm{dB}$ ).

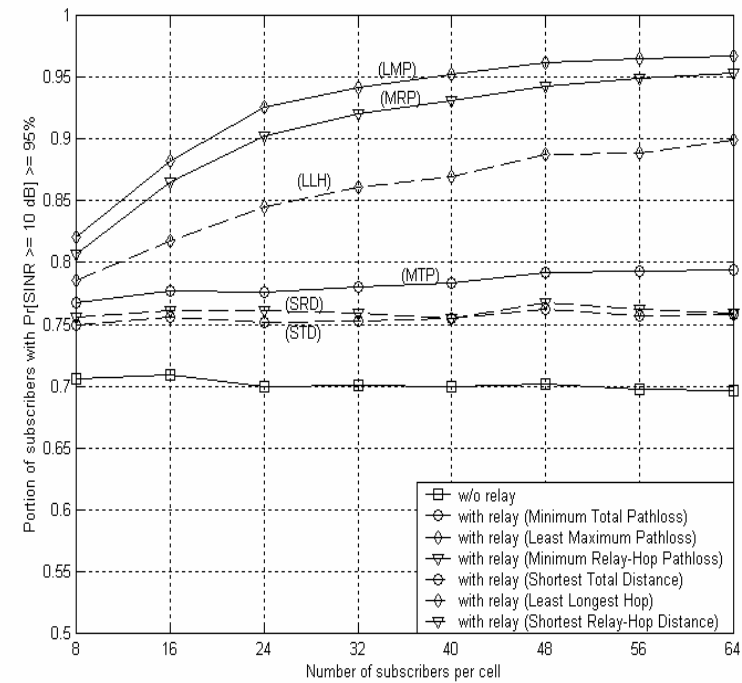

Fig. 2. Coverage vs. number of subscribers per cell (with power control, maximum relay node tx power: $20 \mathrm{~mW}(13 \mathrm{dBm})$ ).

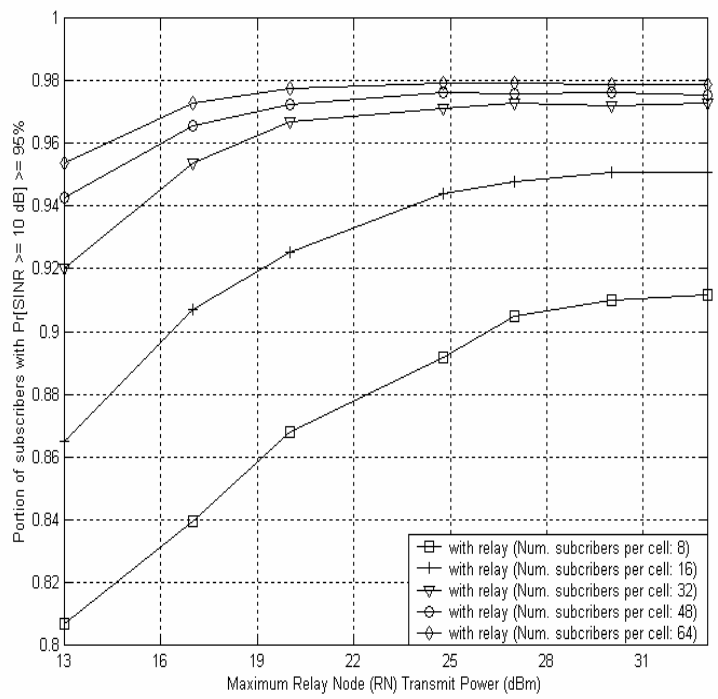

Fig. 3. Coverage vs. maximum relaying node transmit power (with power control, Least Maximum Pathloss (LMP) selection). 